> Les 19 et 20 juin 2019, la conférence internationale sur la recherche dans la dystrophie facio-scapulo-humérale (FSHD) a eu lieu à Marseille. La rencontre rassemblant 180 participants, médecins, scientifiques et patients était organisée au Palais du Pharo à Marseille. Ce site historique emblématique surplombant le Vieux Port et faisant face à Notre Dame de la Garde a été construit dans la seconde moitié du xix ${ }^{e}$ siècle par Napoléon III pour son épouse, l'impératrice Eugénie. <

Depuis 1994, la conférence annuelle organisée par l'association américaine FSH Society a toujours été un moment privilégié pour la communauté des chercheurs et cliniciens travaillant sur la FSHD. Les échanges qui s'y déroulent ont largement contribué à faire avancer la recherche dans cette maladie et permis l'émergence de découvertes fondamentales, tant sur les bases génétiques de la FSHD, les mécanismes moléculaires associés, que sur le développement et le partage de modèles cellulaires et animaux, l'identification de cibles thérapeutiques, et plus récemment, vers le développement d'essais cliniques.

La conférence annuelle FSHD s'est donc tenue exceptionnellement en Europe. Un choix destiné à souligner la contribution majeure de nombreux centres européens de recherche dans la compréhension de cette maladie. Le comité scientifique s'est efforcé de proposer un programme alliant recherche fondamentale et recherche clinique. Ceci afin de renforcer leurs liens mutuels, indispensables dans la perspective de développement de thérapies ciblées. Ces thérapies nécessitent plus que jamais une recherche intégrée partant du patient, une connaissance complète des caractéristiques cliniques de la FSHD, de l'histoire naturelle de la maladie, de sa progression et des corrélations génotype-phénotype pour une meilleure prise en charge.

Le congrès a réuni plus de 180 participants venus des cinq continents. Cinquante-deux présentations orales et plus de trente-cinq communications affichées ont été réparties en dix sessions scientifiques.

\title{
Le colloque \\ annuel de la FSHD Society s'invite à Marseille
}

\author{
Frédérique Magdinier ${ }^{1 *}$, Karine Nguyen ${ }^{1,2}$,
} Shahram Attarian ${ }^{1,3,4}$

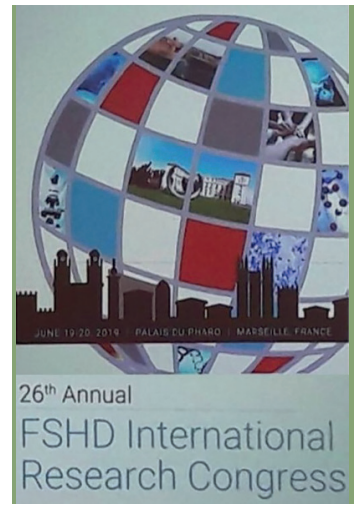

Ces deux journées consacrées à la recherche ont été précédées par la première réunion internationale des associations de patients atteints de FSHD. Cette réunion qui a été résumée par Mark Stone, le directeur général de la FSH Society à l'ouverture du congrès, a été l'occasion de réunir les représentants de patients de onze nations différentes, dont six pays européens mais aussi du Brésil, de Chine, des ÉtatsUnis, d'Israël et du Japon, l'ensemble totalisant treize associations de patients.

L'introduction du congrès a été marquée par le témoignage de deux patients français atteints de myopathie FSH, Pierre Laurian et Marie Martine Fleck. Grâce à une courte vidéo de Pierre Laurian, disponible en ligne, les participants ont pu se plonger au cœur de la vie d'un patient qui livre avec simplicité les difficultés de son quotidien. Les deux intervenants ont ensuite pris la parole à tour de rôle pour livrer à l'assemblée ce que représente le fait de vivre avec une FSHD et la façon dont ils luttent contre leur maladie.

Deux séances plénières ont ouvert la première et la deuxième journée des sessions scientifiques. Lors de la première conférence, le Pr Michel Fardeau a remémoré les travaux pionniers de Landouzy et Dejerine qui ont décrit en 1884 le premier cas «d'atrophie musculaire progressive de l'enfant », comme une affection du système musculaire débutant généralement au cours de l'enfance par une atteinte des muscles de la face, sans altération du système nerveux, et qui doit être dissociée de l'atrophie musculaire progressive myélopathique de l'adulte, travaux publiés en 1885 dans «Revue de médecine » sous le titre de «myopathie atrophique progressive; myopathie sans neuropathie 
FSHD1

Nombre de D4Z4 <11

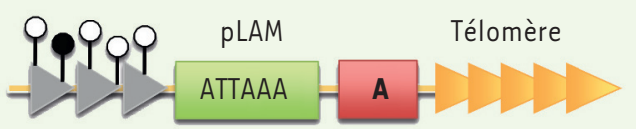

FSHD2

Nombre de D4Z4 >11

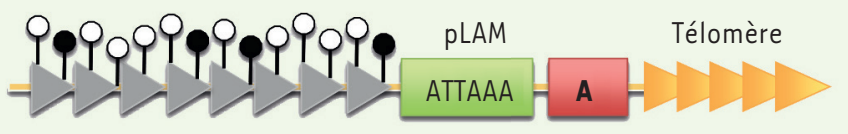

Perte de fonction de SMCHDI

Hypométhylation de l'ADN ; relaxation chromatinienne

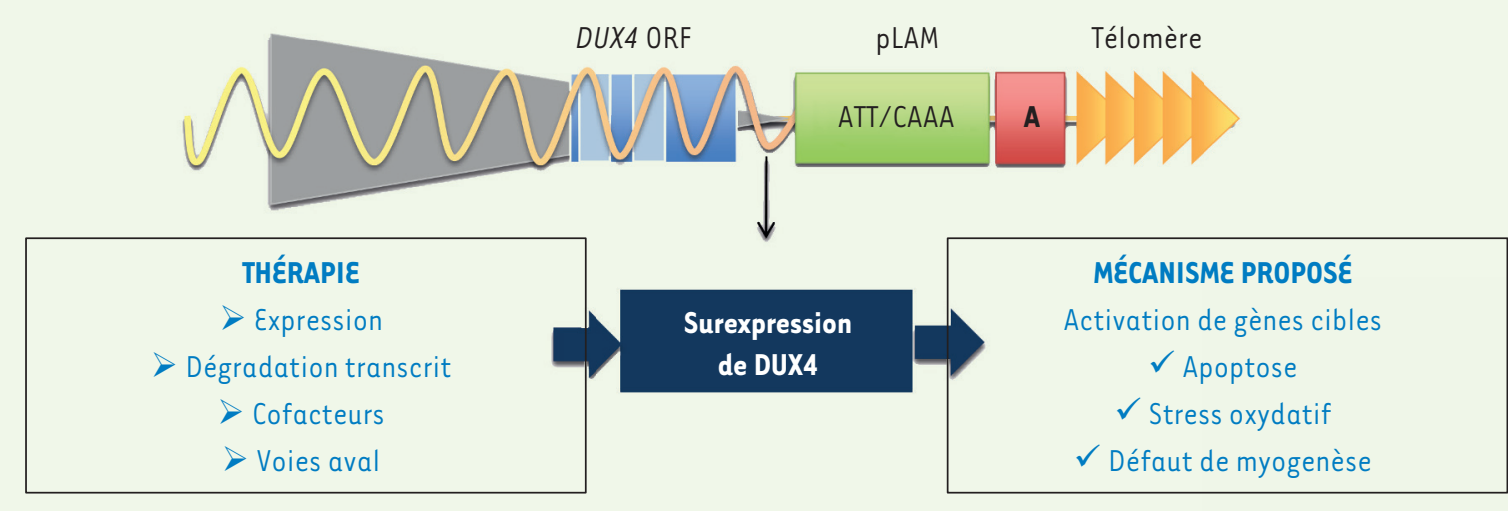

Figure 1. Représentation schématique du locus subtélomérique 4q35. Les répétitions D4Z4 (représentées par des flèches grises) sont situées à une distance d'environ $20 \mathrm{~kb}$ du télomère, à l'extrémité du bras long du chromosome 4q (flèches jaunes). Dans la population générale, le nombre de ces répétitions est supérieur à 10 et varie de 11 à environ 100 . Chez les patients atteints de FSHD1, le nombre de D4Z4 est réduit en dessous d'un seuil de 11 unités. Ce raccourcissement se produit principalement sur les haplotypes de type A, qui sont caractérisés par la présence d'une séquence de 260 bp appelée pLAM contenant un site de polyadénylation. Dans la FSHD2, le nombre de répétitions D4Z4 est supérieur à 11 copies et environ $80 \%$ de ces patients portent une mutation du régulateur chromatinien SMCHD1. La FSHD est caractérisée par une diminution de la méthylation des répétitions $\mathrm{D} 4 \mathrm{Z4}$. Le modèle actuel prévalent pour expliquer la pathogenèse associe cette baisse de méthylation à une relaxation de la chromatine et une surexpression du facteur de transcription DUX4 codé par la dernière répétition. Les conséquences biologiques de surexpression de DUX4 sont indiquées de même que les perspectives thérapeutiques visant à corriger cette expression ectopique et le phénotype musculaire.

débutant d'ordinaire dans l'enfance, par la face » (Landouzy et Dejerine, 1885). L'intervention du Pr Fardeau a été suivie par une présentation du Pr Nicolas Lévy (département de Génétique Médicale du CHU de la Timone) qui a présenté l'état des connaissances concernant la génétique de la FSH, de l'identification du locus $4 q 35$ au début des années 1990 jusqu'à l'identification plus récente du gène SMCHDl et à de nombreux variants du locus $4 q 35$ découverts en particulier grâce à la technique du peignage moléculaire.

Le deuxième jour, Brad Cairns (Utah Medical School), a présenté les travaux novateurs de son équipe sur le rôle physiologique du gène DUX4 comme «activateur du programme d'expression des gènes du zygote ». Cette étape très précoce du développement est nécessaire au déclenchement du programme génétique qui transforme un zygote composé de quatre cellules en embryon au cours des étapes très précoces de l'embryogenèse. De la même façon, M. Percharde (MRC, Imperial College, Londres) a décrit au cours de la session consacrée au rôle de DUX4, le rôle physiologique de ce facteur de transcription chez la souris (Dux) au cours des étapes initiales de l'embryogenèse, au stade 2 cellules chez la souris où Dux active l'expression des rétrotransposons de type Line et la production d'ARNs capables de s'associer à KAPI et à la nucléoline pour supprimer l'expression de Dux.

\section{Phénotype clinique, corrélations génotype- phénotype et épigénétique}

Au cours de la première session dédiée aux aspects cliniques de la maladie, les spécificités du phénotype clinique de la FSHD infantile, l'histoire naturelle de la maladie dans ce sous-groupe de patients, dont $14 \%$ surviennent de novo, les atteintes systémiques et leurs implications dans l'inclusion de ces patients dans des essais cliniques ont été présentés ( $N$. Voermans, Radhoud UMC). J. ljspeert du même centre a montré à l'aide de vidéos comment différentier les patients atteints d'une dyskinésie scapulaire d'origine musculaire d'une atteinte neurologique. S. Sacconi (Université Côte d'Azur) a décrit une augmentation de la fréquence 
de certaines pathologies cutanées dans la FSHD, avec cependant aucune corrélation entre ces atteintes et la sévérité de l'atteinte musculaire. C. Hangül (Akdeniz University) a évalué l'effet des œstrogènes et autres hormones sur les atteintes faciales grâce à un scan 3D du visage et corrélé les niveaux d'atteinte faciale à la sévérité du phénotype et au statut hormonal avec une corrélation significative pour 38 patients. Egalement et afin de tester l'effet des hormones dans cette pathologie, F. Moretti (Rome) a présenté la capacité de régénération de péricytes humains implantés chez la souris et confirmé l'effet protecteur des œstrogènes qu'elle avait décrit in vitro, vis à vis de la toxicité induite par la surexpression de DUX4.

Les difficultés à établir une corrélation génotype-phénotype ont été soulevées lors de plusieurs présentations. M. Kupiers (Leiden University Medical Center) a illustré la difficulté à corréler les différents haplotypes définis comme permissifs avec la maladie. C. Bettio (Université de Modène) a également illustré ce point sur la base d'une analyse de 1703 patients grâce à la classification CCEF (Comprehensive Clinical Evaluation Form) et a montré la grande variabilité phénotypique entre patients avec $45 \%$ des patients ne présentant pas d'atteinte faciale. Comme souligné ensuite par G. Ricci (Université de Modène) et L. Ruggiero (Université Federico II), cette classification, qui doit être validée dans des études longitudinales, peut aider à comprendre la complexité génétique et épigénétique de cette maladie et la variabilité de son histoire naturelle [1].

Faisant suite aux recommandations du congrès de l'ENMC qui s'est tenu fin 2016 [2], K. Mul (Radboud UMC) a dressé un état des lieux des bases de données et des 13 registres nationaux déjà recensés pour la FSH. L'exposé de tous ces travaux souligne la nécessité de prendre en compte l'ensemble des éléments en notre possession pour établir des corrélations génotype-phénotype dans cette pathologie. De plus, l'harmonisation des données recueillis dans les registres devrait permettre à terme une meilleure exploitation des données cliniques et moléculaires, en particulier dans la perspective d'essais cliniques.

De longue date, les changements épigénétiques ont été associés à la FSH (Figure 1). L'analyse de la méthylation d'une large cohorte de patients atteints de FSHDI montre une corrélation entre le nombre de répétitions $D 4 Z 4$ et la méthylation de la séquence de polyadénylation située en aval du dernier D4Z4 (G. Deidda, Institute of Cell Biology and Neurobiology). Dans une étude portant sur 59 individus de neuf familles répartis sur trois générations, P. Lunt (Bristol University) a discuté la possibilité d'une empreinte parentale dans la FSH, avec une survenue plus précoce et une hypométhylation plus marquée lorsque l'anomalie génétique est héritée de la mère. Des mutations de SMCHDI ont été associées à la FSHD de type 2 mais aussi à un autre syndrome sans similitude avec la FSHD, le syndrome arhinie-microphtalmie de Bosma (BAMS). Dans ces deux pathologies, une hypométhylation de D4Z4 a été mise en évidence. RJLF. Lemmers (Leiden University Medical Center) a discuté la nécessité de corréler la présence d'une mutation de SMCHDI à un changement de méthylation de D4Z4 pour la validation fonctionnelle de ces mutants [3]. M. Blewitt (The Walter and Eliza Hall Institute of Medical Research) a présenté la structure cristallographique de la protéine SMCHDI et identifié différents résidus critiques pour l'association de la protéine à l'ADN ou à la chromatine, dont certains mutés chez les patients FSHD2. De plus, parmi les processus épigénétiques associés à la FSHD, J.D. Robin (département de Génétique de Marseille) a montré que l'organisation tridimensionnelle de l'allèle pathogène est altéré dans les cellules de patients FSHD1 et FSHD2 porteurs de mutations de SMCHDI indiquant un lien entre modification épigénétique du locus et formation de boucles chromatiniennes à ce locus [4].

\section{Le phénotype musculaire de la FSHD}

Plusieurs études ont illustré les travaux menés grâce à la résonance magnétique nucléaire (RMN). S.J. Tapscott (Fred Hutchinson Cancer Research Center) a présenté une étude récente basée sur l'utilisation de biopsies à l'aiguille guidées par RMN et une analyse histologique et transcriptomique des tissus prélevés [5]. Cette technique qui permet un suivi longitudinal des patients montre une réponse inflammatoire, des modifications de la matrice extracellulaire et une augmentation des immunoglobulines. Dans une étude pilote chez 22 patients, B. van Engelen (Radhoud UMC, Leiden) a montré une bonne corrélation entre évaluation clinique, section musculaire et sévérité de la maladie. Chez 25 patients, $\varepsilon$. Salort-Campana (CHU de la Timone) a présenté une méthode d'évaluation de l'infiltration adipeuse qui corrèle avec la sévérité de la maladie. Sur la base de ses travaux, elle a présenté une méthode de segmentation semi-automatisée permettant une meilleure évaluation des atteintes musculaires. Dans une $3^{e}$ étude de 30 patients chinois portant sur l'évaluation de huit régions du corps, W. Zhu (Fudan University) a montré une bonne corrélation entre mesures en RMN et l'évaluation de la sévérité.

L'ensemble de ces travaux positionne favorablement la RMN comme un outil visant à déterminer de nouveaux biomarqueurs de la FSHD, pour la caractérisation, le suivi des patients et à terme l'évaluation du bénéfice de thérapies ou en particulier comme l'a illustré M. Montforte (Fondazione Policlinico Gemelli IRCCS) dans les phases actives de progression de la maladie, avant la perte musculaire.

De plus, certaines études fonctionnelles ont révélé que la mesure de force de flexion du genou était un outil de suivi performant, en accord avec des études antérieures suggérant une atteinte précoce des muscles ischiojambiers dans la FSHD. J. Han (University of California Irvine) a décrit un nouvel outil d'évaluation fonctionnelle des membres supérieurs, permettant de mesurer l'évolution de la faiblesse des membres supérieurs en 
fonction de l'amplitude du mouvement des épaules [6]. Cette même équipe a décrit un test fonctionnel mesurant le temps nécessaire au passage de la position en décubitus dorsal à la position assise, utile au suivi des patients. K. Mul a décrit l'élaboration d'une échelle de mesure de 159 mesures d'activité et évalué cette échelle chez 47 individus cliniquement sains et les données cliniques d'environ 500 patients de trois pays (Pays-Bas, France et Royaume-Uni) dans la perspective d'étendre cette échelle à d'autres pays. Ces travaux soulignent la nécessité d'élaboration de tests fonctionnels fiables pour évaluer l'évolution des patients FSHD.

\section{Expression de DUX4, modèles cellulaires et animaux}

Deux sessions ont été consacrées aux travaux dédiés à l'étude des mécanismes de la maladie et plus particulièrement au rôle de DUX4 considéré comme acteur majeur de la survenue de la pathologie. L. Hayward (University of Massachusetts Medical School) a présenté une modélisation in vitro de la myogenèse à partir de cellules souches pluripotentes induites de patients présentant une survenue précoce ou tardive de la maladie qui montre une activation des cibles de DUX4 dans les étapes précoces de la différenciation.

En cherchant à évaluer l'impact du stress oxydatif et génotoxique dans la FSHD, M. Sasaki Honda (Kyoto University) a montré une influence de ceux-ci sur l'expression de DUX4. Egalement, A. Karpukhina (Lomonosov Moscow State University, Russie) a montré que l'induction de DUX4 dans la lignée MB136-iDUX4 de myoblastes humains, se traduit par une augmentation du stress oxydatif et altère le processus de myogenèse. $\varepsilon n$ accord avec ces résultats sur le stress oxydatif, A. de Simone (University of Massachusetts Medical School) a montré que DUX4 induit une délocalisation des mitochondries selon un mécanisme dépendant de l'acide hyaluronique. De plus, réduire l'expression de DUX4 permet de réduire le stress oxydatif et les défauts membranaires des cellules FSHD (y.W. Chen, Children's National Hospital).

M. Panamorova (King's College, Londres) a présenté ses résultats sur la diminution d'un nouveau facteur de transcription et montré que la réexpression de ce facteur était capable de restaurer la prolifération et la différentiation de myoblastes FSHD. A. Greco (Radhoud UMC, Leiden) a montré une augmentation des marqueurs d'inflammation TNF $\alpha$ dans la FSHD, susceptibles de signer une réponse inflammatoire de l'atteinte musculaire. N. Saad (Nationwide Children's Hospital, Columbus) a identifié un micro-ARN capable d'inhiber DUX4 et de limiter les effets délétères de son expression in vitro, sur des cellules mais aussi in vivo, dans un modèle murins d'induction de DUX4.

Plusieurs autres modèles animaux de surexpression de DUX4 ont aussi été présentés. P. Jones (Université de Reno) a décrit le phénotype musculaire d'un modèle d'induction d'expression de DUX4 et l'accumulation de défauts dépendant de cette surexpression. Les travaux présentés par J. Chamberlain (Washington University) utilisant un modèle d'expression de DUX4 placé sous dépendance de son propre promoteur montre un phénotype musculaire caractérisé par la présence de fibres angulaires et ramifiées et un profil d'expression similaire à celui décrit par l'équipe de P. Jones. En étudiant les étapes précédant l'apparition d'un phénotype musculaire après transduction de DUX4 dans des cellules musculaires grâce à un vecteur AAV, P. Gregorevic (University of Melbourne) a montré une baisse de l'activité contractile dans la FSHD, à partir d'organoïdes musculaires.

\section{Développement thérapeutique et essais cliniques}

La mise au point de thérapies dans la FSHD progresse et différents essais cliniques en cours et à venir ont été présentés. La plupart de ces essais ciblent l'expression de DUX4, son niveau de transcrit, ses cofacteurs et voies en aval, ou encore la croissance et la fonction musculaire (Figure 1).

0 . Wallace (Fulcrum Therapeutics) a montré que les inhibiteurs de la p38 MAP kinase suppriment l'expression de DUX4 et empêchent l'apoptose dans les myotubes FSHD. Fulcrum Therapeutics prévoit de réorienter le losmapimod, un inhibiteur p38alpha/beta vers des essais cliniques de phase II pour la FSHD d'ici la fin de l'année. R. Bloch (Maryland University) a présenté les résultats de greffes de cellules humaines dans un modèle murin et la production de muscles humanisés innervés [7]. Dans ce modèle comme dans le modèle présenté par F. Sverdrup (Université de Saint Louis), le phénotype musculaire et l'inflammation associés à la surexpression de DUX4 peuvent être régulés par inhibition de la voie p38 [8]. J. De Maeyer (Facio Therapies) a présenté les résultats de criblage de molécules qui mesurent simultanément l'expression de DUX4 et plusieurs paramètres phénotypiques dans les cellules musculaires primaires. Ce criblage a permis d'identifier les voies $p 38$ et CKI comme voies susceptibles de supprimer l'expression de DUX4.

L. Wallace (Nationwide Children's Hospital, Columbus) a présenté une approche de thérapie anti-DUX4 à base d'ARNs interférents tandis que R. Maruyama (Université d'Alberta), a fait état de la capacité des gapmers modifiés à réduire sélectivement et efficacement les niveaux de transcrits DUX4 dans les myotubes FSHD et le modèle souris FLEX-DUX4.

D. Gabellini (IRCCS San Raffaele Scientific Institute, Milan) a décrit un nouveau processus de régulation de D4Z4 dépendant d'un long ARN non codant, DBE-T et décrit la possibilité de moduler pharmacologiquement cette voie de régulation. De même, CD. Sarsons de la société Resverlogix a décrit une nouvelle classe de modulateurs épigénétiques (BETi) capables de moduler les processus épigénétiques conduisant à l'expression de DUX4 tandis que M. Kyba (University of Minnesota) a rapporté que l'expression de DUX4 entraînait une augmentation massive de l'histone acétylée $\mathrm{H} 3$ et a 
montré qu'un nouvel inhibiteur de l'acétyltransférase p300/CBP inhibait l'expression de DUX4 dans les myoblastes FSHD.

J. Statland (University of Kansas Medical School) a enfin présenté des données sur l'augmentation de la dose dans le cadre d'une étude de phase 2 en cours sur l'ACE-083, un inhibiteur de la myostatine et autres régulateurs négatifs de la croissance musculaire qui montrent une augmentation du volume musculaire et une diminution de la fraction graisseuse musculaire.

\section{Conclusion}

Dans leur grande majorité, les travaux présentés lors de ce colloque illustrent la nécessité d'une caractérisation complète des patients dans leur diversité phénotypique, la nécessité d'identifier des biomarqueurs sensibles pour le suivi longitudinal des patients et l'efficacité des essais cliniques à venir mais aussi la nécessité de se reposer sur des registres harmonisés à l'échelle internationale afin de préparer ces essais cliniques. $\diamond$

Marseille welcomes the FSHD Society International Research

Conference

\section{REMERCIEMENTS}

Le meeting a pu être organisé grâce au soutien financier de Acceleron Pharma, AFM-Telethon, Bulien Family and Friends, Chris Carrino Foundation for FSHD, Filnemus, Friends of FSH Research, FSHD Society, Fulcrum Therapeutics, Genomic Vision, Muscular Dystrophy Association USA, Muscular Dystrophy UK, the U.S. National Institutes of Health, Wellstone Center for FSHD Research, and the University of Nevada Reno School of Medicine.

Nous remercions également le laboratoire Marseille Medical Genetics (MMG, U1251) ; l'Inserm ; l'institut GIPTIS (Genetics Institute for Patients, Therapies,
Innovation and Science) ; le Département de génétique Médicale et le Centre de Référence des maladies neuromusculaires et de la SLA, Hôpital de la Timone, Marseille.

\section{LIENS D'INTÉRÊT}

Les auteurs déclarent n'avoir aucun lien d'intérêt concernant les données publiées dans cet article.

\section{RÉFÉRENCES}

1. Ricci G, Cammish P, Siciliano G, et al. Phenotype may predict the clinical course of facioscapolohumeral muscular dystrophy. Muscle Nerve 2019; 59:711-3.

2. Mul K, Kinoshita J, Dawkins $H$, et al. $225^{\text {th }}$ ENMC international workshop: a global FSHD registry framework, 18-20 November 2016, Heemskerk, The Netherlands. Neuromuscul Disord 2017 ; 27 : 782-90.

3. Lemmers R, van der Stoep N, Vliet PJV, et al. SMCHDl mutation spectrum for facioscapulohumeral muscular dystrophy type 2 (FSHD2) and Bosma arhinia microphthalmia syndrome (BAMS) reveals disease-specific localisation of variants in the ATPase domain. J Med Genet 2019; 56:693-700.

4. Gaillard MC, Broucqsault N, Morere J, et al. Analysis of the $4 q 35$ chromatin organization reveals distinct long-range interactions in patients affected with facio-scapulo-humeral dystrophy. Sci Rep $2019 ; 9$ : 10327

5. Wang LH, Friedman SD, Shaw D, et al. MRI-informed muscle biopsies correlate MRI with pathology and DUX4 target gene expression in FSHD. Hum Mol Genet 2019 ; 28 : 476-86.

6. Hatch MN, Kim K, Kurillo G, et al. Longitudinal study of upper extremity reachable workspace in fascioscapulohumeral muscular dystrophy. Neuromuscul Disord 2019; 29 : 503-13.

7. Mueller AL, O'Neill A, Jones TI, et al. Muscle xenografts reproduce key molecular features of facioscapulohumeral muscular dystrophy. Exp Neurol $2019 ; 320: 113011$.

8. Oliva J, Galasinski S, Richey A, et al. Clinically advanced $p 38$ inhibitors suppress DUX4 expression in cellular and animal models of facioscapulohumeral muscular dystrophy. J Pharmacol Exp Ther 2019; 370 : 219-30.

\section{TIRÉS À PART}

F. Magdinier

\author{
Membres du comité scientifique \\ June Kinoshita, Lexington, USA \& Jamshid Arjomand, San Diego, USA, FSH Society \\ Dr. Frédérique Magdinier, MMG, Marseille ; Pr. George Padberg, Radboud UMC, Nijmegen, Pays Bas
}

\title{
Sessions scientifiques et modérateurs des sessions
}

Session 1 : The FSHD clinical phenotype. George W. Padberg, Nijmegen, Pays Bas \& Peter Lunt, Bristol, Royaume Uni Session 2 : Genetics and molecular findings for genotype-phenotype correlations and genetic diagnostics. Rossella Tupler, Modena, Italie \& Meena Upadhyaya, Cardiff, Royaume Uni

Session 3 : Molecular mechanisms, DUX4, downstream targets, other players. Peter Zammit, London, Royaume Uni \& Michael Kyba, Minneapolis, USA

Session 4 : Molecular mechanisms and animal models. Alexandra Belayew, Mons, Belgique \& Scott Q. Harper, Columbus, USA Session 5 : DNA methylation and epigenetics. Marnie Blewitt, Melbourne, Australie \& Frédérique Magdinier, Marseille, France Session 6 : Muscle pathology and imaging. Giorgio Tasca, Rome, Italie \& Rabi Tawil, Rochester, USA

Session 7 : Natural History. Sabrina Sacconi, Nice, France \& Karlien Mul Nijmegen, Pays Bas.

Session 8 : Registries. Sabrina Sacconi, Nice, France

Session 9 : Therapeutic intervention. Stephen J. Tapscott, Seattle, USA \& Fran Sverdrup, St. Louis, USA

Session 10 : Clinical evaluation, outcome measures and clinical trial readiness. Jeffrey Statland, Kansas City, USA \& Baziel van Engelen Nijmegen, Pays Bas. 